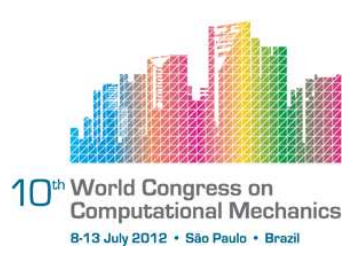

\title{
NOVEL BASIS FUNCTIONS FOR THE PARTITION OF UNITY BOUNDARY ELEMENT METHOD FOR HELMHOLTZ PROBLEMS
}

\author{
M. J. Peake, J. Trevelyan, G. Coates
}

School of Engineering and Computing Sciences, Durham University (m.j.peake@ durham.ac.uk)

\begin{abstract}
The boundary element method (BEM) is a popular tool for wave scattering problems. To reduce the number of degrees of freedom required, the partition of unity BEM (PU$B E M)$ was developed in which the approximation space is enriched with a linear combination of plane-waves. Recent work has shown that the element ends are more susceptible to errors in the approximation than the mid-element regions. In this paper we propose that this is due to the reduced order of continuity in the Lagrangian shape function component of the basis functions. It will demonstrated that choosing trigonometric shapes functions, rather than classical quadratic shape functions, provides accuracy benefits.
\end{abstract}

Keywords: Partition of unity, BEM, shape functions, Helmholtz, wave scattering.

\section{INTRODUCTION}

The boundary element method (BEM) was first used to solve the Helmholtz equation by Banaugh and Goldsmith [1]. Since then, the BEM has become a well-established technique for wide range of problems. In particular, the BEM's formulation is useful for problems with infinite domains as it automatically satisfies boundary conditions at infinity and no domain truncation is required.

Babuŝka and Melenk developed the partition of unity method [2] in which, for acoustics, the approximation domain may be enriched by a linear combination of plane waves. This was developed for the FEM by Laghrouche et al. [3], for the collocation BEM by PerreyDebain et al. [4], and for the Galerkin BEM by Bériot et al. [5]. The partition of unity boundary element method (PU-BEM) significantly reduces the number of degrees of freedom per wavelength, $\tau$, required for a prescribed error.

Trevelyan and Coates [6] presented an adaptive basis for the collocation PU-BEM. They noted that residual errors were largest at the ends of elements. It was suggested that this was due to the lack of continuity in the quadratic shape functions used. This paper will introduce a novel set of shape functions that provide greater continuity between elements and, thus, reduce these residual errors. 


\section{PARTITION OF UNITY BOUNDARY ELEMENT METHOD}

Let $\Omega \subset \mathbb{R}^{2}$ be a domain, with no exterior boundary and with a smooth internal scatterer of boundary $\partial \Omega=\Gamma$. In the frequency domain, propagation of sound waves is governed by the Helmholtz equation,

$$
\nabla^{2} \phi(\mathbf{q})+k^{2} \phi(\mathbf{q})=0, \quad \mathbf{q} \in \Omega,
$$

where $\nabla^{2}$ is the Laplacian operator, $\phi \in \mathbb{C}$ is a wave potential, and $k$ is the wavenumber.

The derivation of the classical, polynomial basis BEM for (1) is well known [7]. Using this method, the wave potential on the boundary of a scatterer is written as

$$
\phi^{e}(\mathbf{q})=\sum_{j=1}^{J} N_{j}(\xi) \phi_{j}^{e},
$$

where $\phi^{e}$ is the potential at q, described by the local coordinate $\xi$ on element $e, J$ is the number of shape functions, $N_{j}$ is the $j$ th shape function and $\phi_{j}$ is its associated potential. In the PU$\mathrm{BEM}$, the approximation space is enriched by a linear combination of plane waves at each element node, thus the potential on the boundary of a scatterer is expressed as

$$
\phi^{e}(\mathbf{q})=\sum_{j=1}^{J} N_{j}(\xi) \sum_{m=1}^{M} A_{j m}^{e} \exp \left(i k \mathbf{d}_{j m}^{e} \cdot \mathbf{q}\right), \quad\left|\mathbf{d}_{j m}^{e}\right|=1,
$$

where $M$ is the number of plane wave basis functions per node on the element, $\mathbf{d}_{j m}^{e} \in \mathbb{R}^{2}$ are the prescribed directions of the plane waves in the basis, and $A_{j m}^{e} \in \mathbb{C}$ are their amplitudes which are sought as the solution to the BEM system.

$M$ may be chosen such that a prescribed $\tau$ is satisfied, locally and globally. For FEM and BEM approximations, $\tau \geq 10$ is, generally, required; however, it has been shown that, for the PU-BEM, $\tau \simeq 3$ is sufficient for an accuracy $\sim 1 \%$ [4].

\section{TRIGONOMETRIC SHAPE FUNCTIONS}

Quadratic shape functions are commonly used in the both the FEM and BEM; however, no study of shape functions has been carried out for the PU-BEM.

Quadratic shape functions only provide $C^{0}$ continuity. Here, we propose a novel set of shape functions, using trigonometric functions, which provide $C^{1}$ continuity; in some cases, it is possible to obtain $C^{\infty}$. For a 3-noded, continuous element, these shape functions are:

$$
\begin{gathered}
N_{1}(\xi)=-\frac{1}{4} \cos (\pi \xi)-\frac{1}{2} \sin \left(\frac{\pi}{2} \xi\right)+\frac{1}{4}, \\
N_{2}(\xi)=\frac{1}{2} \cos (\pi \xi)+\frac{1}{2}, \\
N_{3}(\xi)=-\frac{1}{4} \cos (\pi \xi)+\frac{1}{2} \sin \left(\frac{\pi}{2} \xi\right)+\frac{1}{4} .
\end{gathered}
$$

These shape functions can be seen in Figure 1a alongside the traditional quadratic shape functions (Figure 1b). The added continuity of these new shape functions can be observed at the element ends where they have zero gradient, giving $C^{1}$ continuity. If adjoined elements are the same length, $C^{\infty}$ continuity is obtained. 


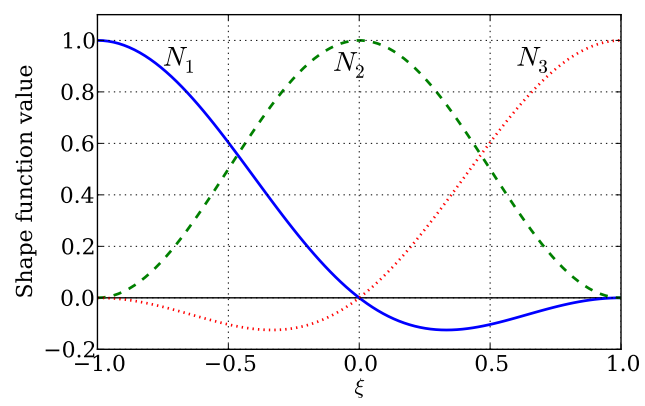

(a) Quadratic shape functions

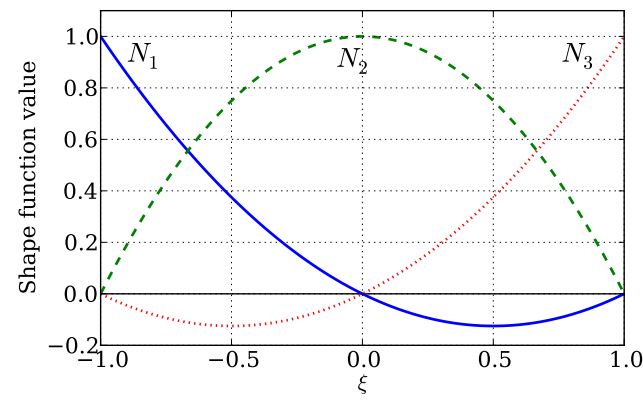

(b) Trigonometric shape functions

Figure 1. Shape functions for 3-noded element with local coordinate $\xi \in[-1,1]$

\section{NUMERICAL RESULTS}

\subsection{Scattering by a circular cylinder}

Consider a cylindrical scatterer, of unit radius, impinged by a unit-amplitude, incident plane wave propagating in the direction $\mathbf{d}^{\mathrm{I}}$. The boundary condition, $\partial \phi / \partial n=0$, is chosen, where $n$ is the outward pointing, unit normal; this is known as the sound-hard condition. The analytical solution for the total field is known [8]. Errors of simulations, $\mathcal{E}$, are calculated using $L_{2}$ norms:

$$
\mathcal{E}=\frac{\left\|\Phi-\Phi^{\mathrm{ex}}\right\|_{L_{2}}}{\left\|\Phi^{\mathrm{ex}}\right\|_{L_{2}}}
$$

where $\Phi$ are potentials calculated from the PU-BEM, and $\Phi^{\mathrm{ex}}$ are potentials calculated analytically.

Figure 2 shows a comparison of errors from PU-BEM simulations, of this problem, using trigonometric and quadratic shape functions over a range of wavelengths. CHIEF points were used to overcome the nonuniqueness problem [9] and the system matrices were solved using singular value decomposition.

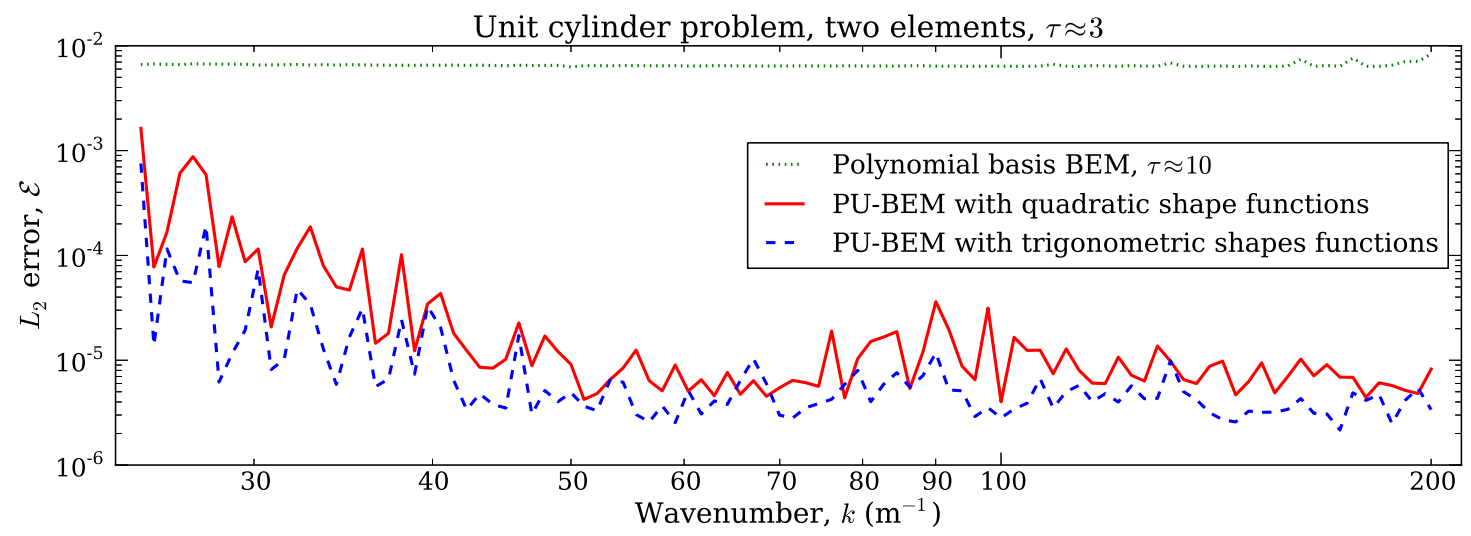

Figure 2. Errors analysis for the hard, unit-radius, cylindrical scatter problem: incident wave $\mathbf{d}^{\mathrm{I}}=(1,0)$

Figure 2 shows clearly that trigonometric shape functions provide an accuracy benefit; however, a plot of the errors over the surface of the cylinder is required to demonstrate where 
these accuracy improvements originate. Figure 3 shows a comparison of the errors arising from each type of shape function; $s \in[0,1]$ is a local coordinate that runs clockwise around the entire cylinder starting from the cartesian coordinate $(1,0)$. Using quadratic shape functions, the error peaks are prominent at the end of the two elements. Using the trigonometric shape functions has significantly reduced the magnitude of these errors.
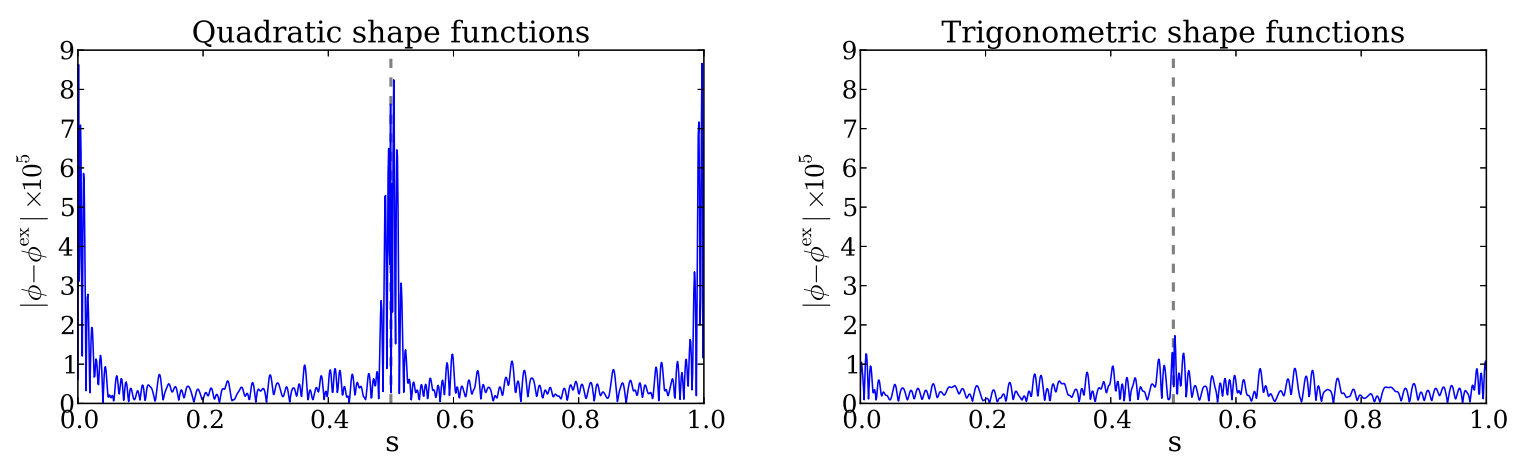

Figure 3. Error, $\left|\phi-\phi^{\text {ex }}\right|$, over surface of circular cylinder problem, $k=50$

\subsection{Scattering by five cylinders}

Consider a set of sound-hard, cylindrical scatterers of arbitrary radii, centred at arbitrary coordinates, impinged by a unit-amplitude, incident plane wave of direction $\mathbf{d}^{\mathrm{I}}$. Linton and Evans [10] published an analytical solution for this problem, by means of an infinite series. PU-BEM simulations were run, using quadratic and trigonometric shapes functions, for the problem of five, sound-hard, unit-radius cylinders equally spaced at $r=3$ from the origin. This problem is chosen because it contains internal reflections between cylinders; this can be seen in the real part of the potential solution, plotted in Figure 4.
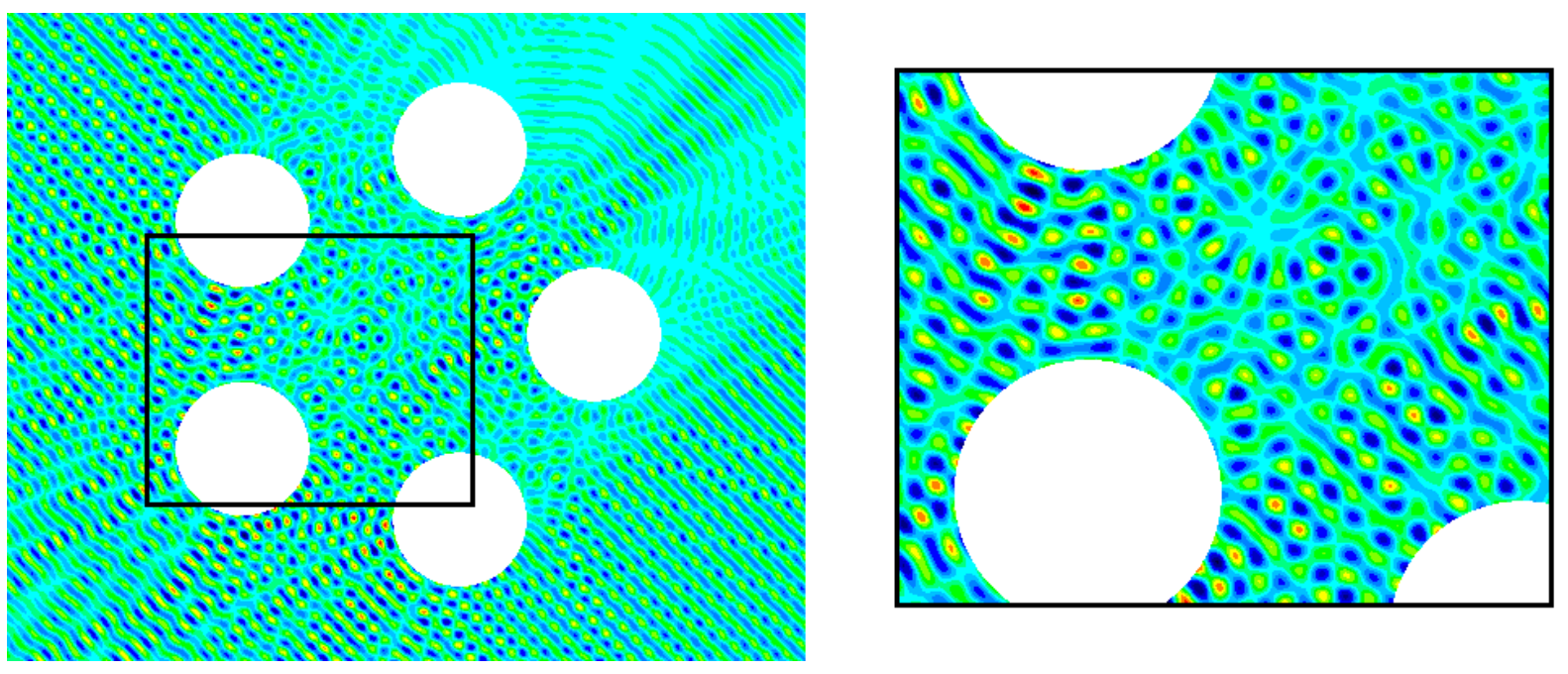

Figure 4. Illustration of the internal reflections caused by the five-cylinder geometry: $\lambda=0.25$

Figure 5 shows the errors, $\mathcal{E}$, from using both types of shape function, over a range of wavelengths. It is clear that the trigonometric shape functions provide an accuracy benefit for the majority of simulations. This is because of the increased continuity, of the shape functions, between elements. 


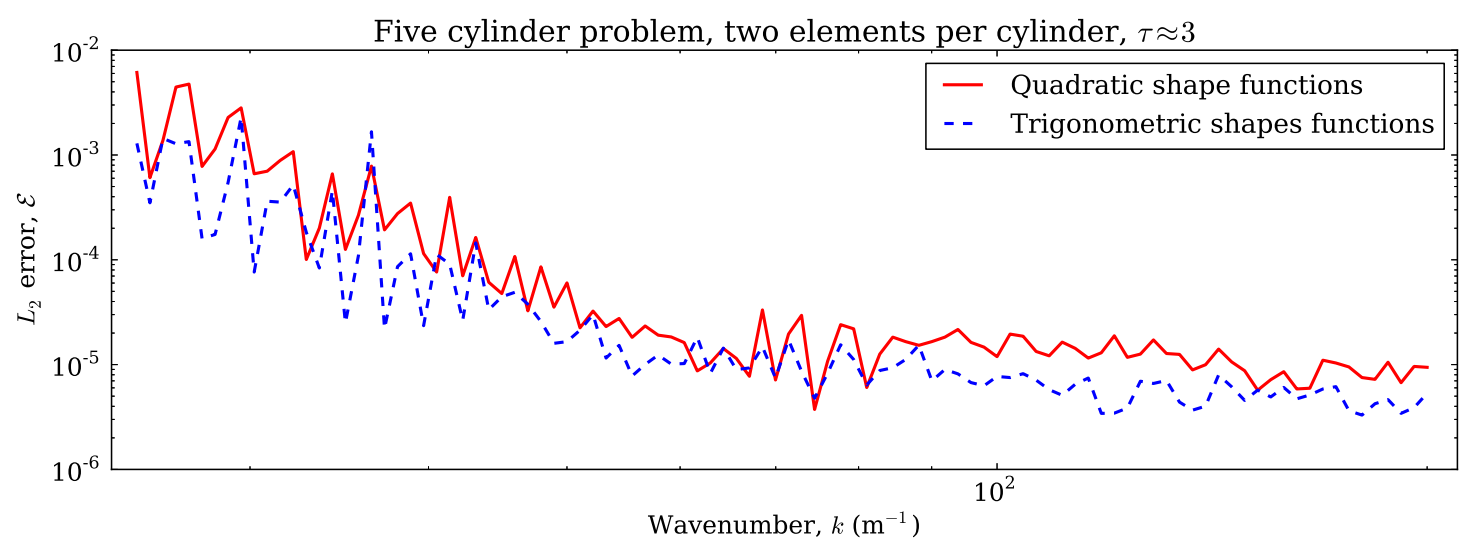

Figure 5. Error analysis for the five-cylinder scatterer problem: incident wave $\mathrm{d}^{\mathrm{I}}=$ $(1 / \sqrt{2}, 1 / \sqrt{2})$

\subsection{Scattering by a capsule}

Scattering by a capsule introduces a $C^{1}$ geometry (see Figure 6). Regions where lines and arcs blend together are susceptible to errors. To investigate the ability of trigonometric shape functions to capture the effect of these this type of boundary, the capsule in Figure 6 was devised; elements of equal length (i.e. elements ends at $s=0,1 / 3,2 / 3$ ) were used to maximise the continuity of the shape functions. As all geometry points are evaluated analytically, this does not affect the accuracy of evaluation of the integration kernels.

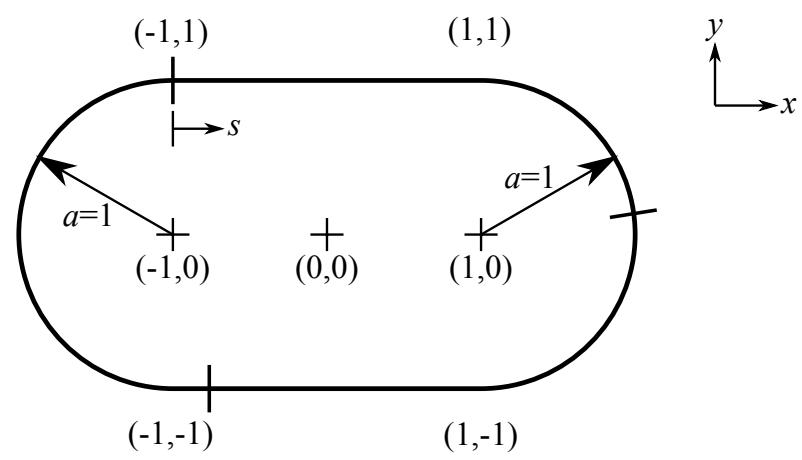

Figure 6. Capsule discretised by three equal-length elements

Figure 7 shows the errors, $\mathcal{E}$, from using both types of shape function, over a range of wavelengths; exact solutions, $\Phi^{\mathrm{ex}}$, were evaluated using a converged method of fundamental solutions (MFS) approach. The trigonometric shape functions produce a clear accuracy benefit at lower wavenumbers; however, at higher wavenumbers, the benefits are reduced. This is because, at high wavenumbers, there are a large number of plane waves in the expansion which become the most dominant part of the basis, i.e. the observable effect of the shape functions is reduced.

Figure 8 shows the absolute difference, along the surface of the scatterer, between the PU-BEM and converged MFS solution. Significant errors at the element ends (denoted by the dashed lines) have been reduced by the trigonometric shape functions. Significant errors at the blend points between the lines and arcs have also been reduced; however, these errors are 
still large in comparison to the errors over the rest of the boundary. The trigonometric shape functions, though continuous through these points, are not sufficient to describe, ideally, the effect the geometry has on the wave potential in those areas.

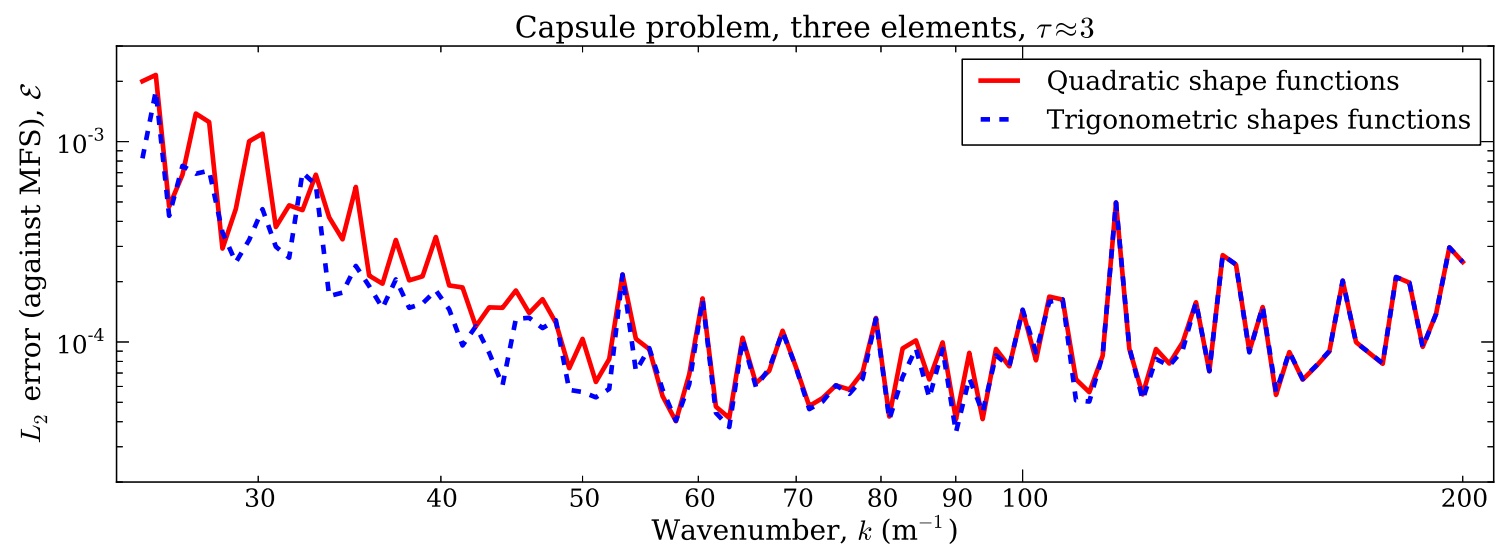

Figure 7. Error analysis for the hard capsule problem: $\mathbf{d}^{\mathrm{I}}=(0.5, \sqrt{3} / 2)$
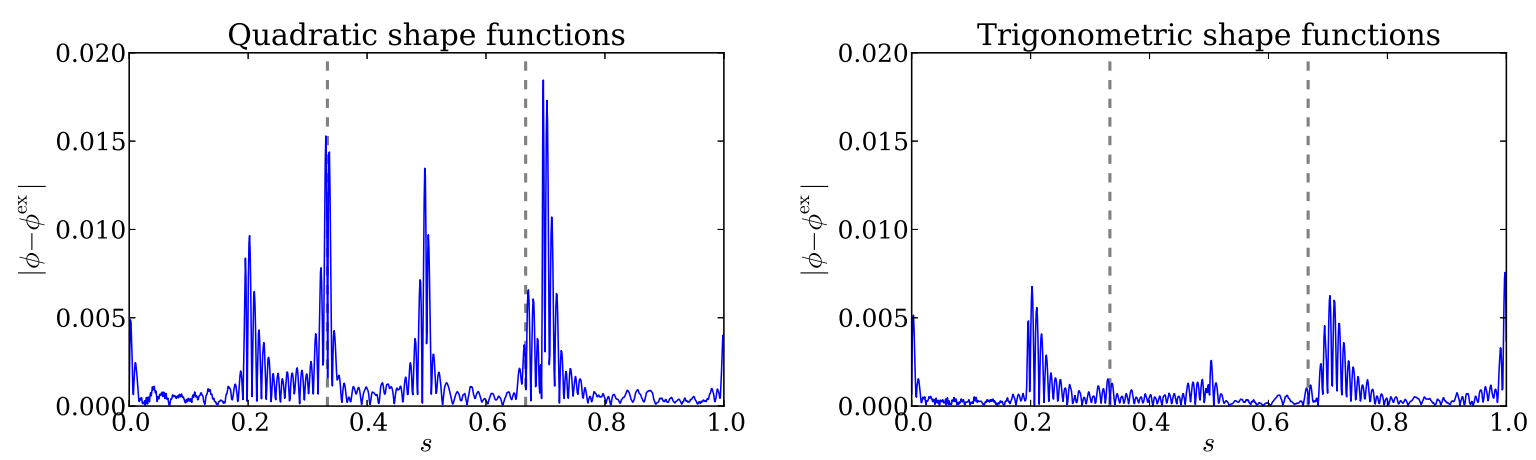

Figure 8. Error, $\left|\phi-\phi^{\mathrm{ex}}\right|$, over surface of capsule problem, $k=25$

\section{CONCLUSIONS}

Using the PU-BEM for wave scattering simulations, errors are found to be at a maximum at the element ends. This is due to a lack of continuity, at the element ends, associated with Lagrangian shapes functions. Trigonometric shape functions increase the continuity at the element ends and, thereby, improve the approximation of potential of such problems. It should be noted that these accuracy gains are not replicable for piecewise quadratic BEM simulations.

For geometries with $C^{1}$ continuity, the PU-BEM is susceptible to errors at geometry blend points. Though trigonometric shape functions do provide an accuracy benefit, more continuity is required. One possible approach is to use non-uniform rational B-splines (NURBS) which can represent circular arcs analytically. 


\section{REFERENCES}

[1] Banaugh R. P., Goldsmith W., "Numerical diffraction of steady acoustic waves by surfaces of arbitrary shape". J. Acoust. Soc. Am. 35(10), 1590-1601, 1963.

[2] Babuŝka I., Melenk J. M., "The partition of unity method". Int. J. Numer. Meth. Engng. 40(4), 727-758, 1997.

[3] Laghrouche O., Bettess P., Astley P., "Modelling of short wave diffraction problems using approximating systems of plane waves". Int. J. Numer. Meth. Eng. 54(10), 1501-1533, 2002.

[4] Perrey-Debain E., Trevelyan J., Bettess P., "Plane wave interpolation in direct collocation boundary element method for radiation and wave scattering: numerical aspects and applications". J. Sound Vib. 261(5), 839-858, 2003.

[5] Bériot E., Perrey-Debain E., Ben Tahar M., Vayssade C., "On a Galerkin wave boundary element formulation for scattering by non-smooth obstacles". Proc. WAVES 2007 Conf. Reading. 400-402, 2007.

[6] Trevelyan J., Coates G., "On adaptive definition of plane wave basis for wave boundary elements in acoustic scattering: the 2D case". Comput. Model. Eng. Sci. 55(2), 147-168, 2010 .

[7] Wrobel L. C., The boundary element method : applications in thermo-fluids and acoustics. John Wiley \& Sons, 2002.

[8] Jones D. S., Acoustic and electromagnetic waves. Clarendon Press, 1986.

[9] Schenck H. A., "Improved integral formulation for acoustic radiation problems". J. Acoust. Soc. Am. 44(1), 41-58, 1968.

[10] Linton C. M., Evans D. V., "The interaction of waves with arrays of vertical circular cylinders". J. Fluid Mech. 215, 549-569, 1990. 